\title{
A Heuristic based on Fuzzy Inference Systems for Multiobjective IMRT Treatment Planning
}

\author{
Joana Dias ${ }^{1,2}$, Humberto Rocha $^{1,2}$, Tiago Ventura $^{3}$, Brígida Ferreira ${ }^{4}$, Maria do Carmo \\ Lopes $^{3,5}$ \\ ${ }^{1}$ CeBER and Faculdade de Economia, Universidade de Coimbra, 3004-512 Coimbra, Portugal \\ ${ }^{2}$ Inesc-Coimbra, Rua Sílvio Lima, Pólo II, 3030-290 Coimbra, Portugal \\ ${ }^{3}$ Serviço de Física Médica, IPOC-FG, EPE, 3000-075 Coimbra, Portugal \\ ${ }^{4}$ School for Allied Health Technologies, Porto, Portugal \\ ${ }^{5}$ I3N, Departamento de Física, Universidade de Aveiro, 3810-193 Aveiro, Portugal \\ joana@fe.uc.pt, hrocha@mat.uc.pt, \\ tiagoventuradipocoimbra.min-saude.pt,brigida@ua.pt, \\ mclopes@ipocoimbra.min-saude.pt
}

\begin{abstract}
Radiotherapy is one of the treatments used against cancer. Each treatment has to be planned considering the medical prescription for each specific patient and the information contained in the patient's medical images. The medical prescription usually is composed by a set of dosimetry constraints, imposing maximum or minimum radiation doses that should be satisfied. Treatment planning is a trial-and-error time consuming process, where the planner has to tune several parameters (like weights and bounds) until an admissible plan is found. Radiotherapy treatment planning can be interpreted as a multiobjective optimization problem, because besides the set of dosimetry constraints there are also several conflicting objectives: maximizing the dose deposited in the volumes to treat and, at the same time, minimizing the dose delivered to healthy cells. In this paper we present a new multiobjective optimization procedure that will, in an automated way, calculate a set of potential nondominated treatment plans. It is also possible to consider an interactive procedure whenever the planner wants to explore new regions in the non-dominated frontier. The optimization procedure is based on fuzzy inference systems. The new methodology is described and it is applied to a head-and-neck cancer case.
\end{abstract}

Keywords: Multiobjective, Radiotherapy Planning, Fuzzy Inference Systems.

\section{$1 \quad$ Introduction}

Radiotherapy is one of the possible treatments used against cancer, possibly combined with surgery and chemotherapy. In a radiotherapy treatment (RT), the patient is immobilized in a couch, and receives radiation from a linear accelerator, mounted on a gantry that can rotate along a central axis parallel to the couch. The rotation of the couch and gantry allows radiation to be delivered from almost any direction (angle) around the tumor. However, the equidistant coplanar angle configuration (radiation beams equally spaced lay on the plane of rotation of the linear accelerator) is usually 
used. There are different RT modalities, sharing essentially the same workflow. First all volumes to treat (Planning Target Volumes - PTV) and radiosensitive structures to spare (Organs at Risk - OAR) are delineated using the patient's 3D medical images. Then, the medical prescription is defined, imposing lower and/or upper radiation doses to be deposited, or maximum /minimum volumes that should receive a given maximum/minimum radiation dose. This medical prescription has then to be translated into a plan configuration. In the last stage, the quality of the proposed treatment plan is analyzed through dose-volume statistic tools and dose distribution inspection. The present work is focused on the process that leads to a treatment plan to be delivered to the patient. In current clinical practice, this process is done by resorting to a computer assisted trial-and-error time consuming procedure using dedicated dose calculation software (Treatment Planning System-TPS). TPS asks the planner to introduce weights, bounds or other parameters. With these parameters fixed, the TPS will run an optimization procedure, generating a dose distribution that will be compared with the desired dose distribution defined by the medical prescription. The planner will iteratively change the TPS dependent parameters, trying to comply with the medical prescription. The procedure is repeated until the planner is satisfied, runs out of time, or runs out of ideas on how to improve the treatment plan. Depending on the complexity of the case, this interactive process can take from several hours to several days for a single patient, and the optimality of the solution is not guaranteed. Moreover, the planner will have to deal with many difficult decisions and tradeoffs. It is not possible to guarantee that a solution satisfying all the dosimetry constraints even exists. If this is the case, the planner will have to try to satisfy the constraints "as much as possible", being difficult to define in a rigorous way this concept. The planner will also have to consider the existence of tradeoffs between the doses delivered to different structures, since RT planning is inherently a multiobjective problem: the maximization of the dose delivered to PTV versus minimization of the dose in OAR.

In this work, Intensity Modulated Radiation Therapy (IMRT) is considered, although the developed methodology can be easily extended to other RT modalities. In IMRT the head of the linear accelerator is composed of pairs of individual leaves that can move independently (multileaf collimator). These leaves will block radiation, and different configurations allow the conformal shaping of the treatment beams to the tumor shape and the possibility of having different radiation intensity profiles. Each radiation beam is interpreted as a set of individual beamlets. In clinical practice, the planner will usually determine a priori the number of beams to use and their directions. For each set of TPS parameters, an optimization procedure is run (IMRT Fluence Map Optimization - FMO) that will generate the optimal radiation intensity associated with each beamlet from each of the angles to be used in the treatment (fluence maps). The dose deposited in each voxel (measured in Gy) can then be calculated. In this paper, we present an approach where the trial-and-error procedure is replaced by an automated procedure that optimizes fluence maps by using Fuzzy Inference Systems (FIS). The procedure will consider different sets of angles, and will calculate a set of potential non-dominated solutions that can then be presented to the planner. Solutions are called "potential" non-dominated because it is not possible to know for sure if they are indeed Pareto optimal solutions. The presented methodology can thus be seen as a heuristic procedure. The paper is organized as follows: in Sec- 
tion 2, a brief review of the literature is presented, focusing on fuzzy logic and multiobjective approaches applied to RT planning. Section 3 describes the mathematical optimization problem and the FMO problem. Section 4 describes the developed approach. Section 5 describes an application to a head-and-neck cancer case. Section 6 presents some conclusions and directions for future research.

\section{Brief review of the literature}

The rules that guide the planner in the interactive process of changing TPS dependent parameters can be hard to represent in a mathematical formal way. They usually are simple rules that can be written in natural language, and one of the ways of representing this kind of information is resorting to fuzzy numbers and fuzzy logic. The methodology presented in this paper is an adaptation of an algorithm previously developed by the authors [1]. All the model parameters are iteratively and automatically changed by resorting to a FIS system, without any type of human intervention. The algorithm considers how far the present treatment plan is from what is desired by the medical prescription, and uses common-sense rules of the form "if the spinal cord is not being spared enough then increase the importance of this structure in the optimization process", translated into fuzzy rules, to automatically tune the TPS parameters. One of the drawbacks of the methodology is that it asks the planner to define priorities associated with each structure (that can all have the same value), calculating a single solution based on those priorities. The method is capable of delivering high quality plans within reasonable computational times. Fuzzy logic has been applied to RT planning before. Li and Yin [2] apply fuzzy logic for determining the best prescription for the normal tissue. Yan et al $[3,4]$ consider the changing of weights assigned to each structure through the use of a FIS composed of eight rules. The authors extend this work [5] by developing a neuro-FIS using a trained neural network to determine the parameters of the fuzzy inference system.

The multiobjective inherently nature of RT planning problems have been recognized by several different authors. It has been demonstrated that multiobjective optimization can help planners, especially the less trained ones, to improve the quality of the treatment plans, with a reduction of planning time [6, 7]. Romeijn et al. [8] present several results showing that under some conditions several non-convex objectives usually used in RT planning can be transformed in convex ones, preserving the set of non-dominated treatment plans. In $[9,10]$ a database of treatment plans is created for a posteriori navigation, under the condition that the multiobjective optimization problem is convex. In [11] the authors analyze two different navigation algorithms, and conclude that only a limited number of plans is needed during navigation. In [12] the authors tackle the problem of non-convexity whenever different sets of beam angles are considered, by developing a methodology that allows the navigation between different convex Pareto surfaces. Teichert et al. [13] present a methodology to compare two convex Pareto sets considering two different sets of beam angles. Metaheuristics have also been applied. Holdsworth et al. $[14,15]$ present a hierarchical evolutionary algorithm for IMRT plan generation. The higher level population represents 
parameters that are used in the fitness function calculation for the lower level deterministic optimization algorithm. Aubry et al. [16] present a simulated annealing approach, where different objective functions are iteratively chosen to guide the algorithm. Lexicographic approaches to radiotherapy planning have been developed [17], considering a pre-determined ordered list of objectives and constraints.

\section{Multiobjective optimization problem}

The multiobjective optimization problem is determined by the medical prescription. The defined constraints are inherently linked to the desired objectives. The type of restrictions and objectives to consider will be patient dependent, but they usually consist of dose-volume restrictions that relate the dose delivered with the volume that receives that dose and that one wishes to maximize or minimize, according to the specific structure. One of the main tools to assess the quality of a RT plan is the Dose Volume Histogram (DVH), so including in the optimization process restrictions and objectives related with points in the DVH has several advantages. However, these type of constraints are usually considered as being very difficult to include in FMO problems [18-20], because they present the drawback of creating a non-convex feasibility space, with many local minima. It can also be useful to consider the mean-taildose rather than conventional dose-volume constraints [21] (mean dose of a hottest or coldest fractional volume). Consider the medical prescription defined in Table 1.

Table 1. Prescribed doses for each structure considered

\begin{tabular}{|c|c|c|c|}
\hline Structure & Type of constraint & & Limit \\
\hline Spinal cord & Maximum dose & Lower than & 45 Gy \\
\hline Brainstem & Maximum dose & Lower than & $54 \mathrm{~Gy}$ \\
\hline Left parotid & Mean dose & Lower than & $26 \mathrm{~Gy}$ \\
\hline Right parotid & Mean dose & Lower than & $26 \mathrm{~Gy}$ \\
\hline $\mathrm{PTV}_{70}$ & $D_{95 \%}$ & Greater than & $66.5 \mathrm{~Gy}$ \\
\hline $\mathrm{PTV}_{70}$ & Maximum dose & Lower than & $74.9 \mathrm{~Gy}$ \\
\hline $\mathrm{PTV}_{59}$ & $D_{95 \%}$ & Greater than & 56.4 Gy \\
\hline $\mathrm{PTV}_{59}$ & $V_{107 \%}$ & Lower than & $\begin{array}{l}\text { Percentage of } \mathrm{PTV}_{70} \text { volume inside } \mathrm{PTV}_{59} \text { plus } \\
\text { a } 10 \% \text { margin }\end{array}$ \\
\hline Body & Maximum dose & Lower than & 80 Gy \\
\hline
\end{tabular}

This medical prescription considers five structures that should be spared (spinal cord, brainstem, left and right parotids, body), and two PTVs that have different dose requirements: $\mathrm{PTV}_{59}$ that should receive $59.4 \mathrm{~Gy}$ and $\mathrm{PTV}_{70}$ that should receive $70 \mathrm{~Gy}$ (the prescribed doses). In this particular case, $\mathrm{PTV}_{70}$ is inside $\mathrm{PTV}_{59}$. In an ideally situation $100 \%$ of $\mathrm{PTV}_{70}$ voxels would receive $70 \mathrm{~Gy}$, and $100 \%$ of $\mathrm{PTV}_{59}$ voxels (except those belonging to $\mathrm{PTV}_{70}$ ) would receive $59.4 \mathrm{~Gy}$. It is not possible to guarantee this complete coverage, so different types of constraints are imposed. This medical prescription can be interpreted as defining the following set of constrains:

- No voxel belonging to the spinal cord should receive more than $45 \mathrm{~Gy}$;

- No voxel belonging to the brainstem should receive more than 54Gy; 
- The mean dose in the parotids should not exceed 26Gy;

- 95\% of the voxels in $\mathrm{PTV}_{70}$ should receive at least $66.5 \mathrm{~Gy}\left(D_{95 \%}\right)$;

- No voxel belonging to $\mathrm{PTV}_{70}$ should receive more than $74.9 \mathrm{~Gy}$;

- 95\% of the voxels in $\mathrm{PTV}_{59}$ should receive at least $56.4 \mathrm{~Gy}\left(D_{95 \%}\right)$;

- The percentage of voxels in $\mathrm{PTV}_{59}$ that are allowed to receive more than $107 \%$ of the prescribed dose $\left(V_{107 \%}\right)$ are limited to the percentage of $\mathrm{PTV}_{70}$ volume inside PTV $_{59}$ plus a $10 \%$ margin.

These constraints are related with optimization objectives:

- The maximum dose received by the spinal cord should be as low as possible;

- The maximum dose received by the brainstem should be as low as possible;

- The mean dose received by the parotids should be as low as possible;

- $D_{95 \%}$ for PTV 70 should be as close to $70 \mathrm{~Gy}$ as possible;

- $D_{95 \%}$ for $\mathrm{PTV}_{59}$ should be as close to $59 \mathrm{~Gy}$ as possible;

- The percentage of voxels in $\mathrm{PTV}_{59}$ that receive more than $107 \%$ of the prescribed dose $\left(V_{107 \%}\right)$ should be minimized.

It is not expected that a single treatment plan will be able to simultaneously optimize all these objectives. If we consider that the set of beam angles is fixed a priori then the multiobjective problem that has to be solved is the FMO problem considering simultaneously several objectives. Let $V$ represent the number of voxels, $N$ the number of beamlets and $D$ the dose matrix, such that $D_{i j}$ represents the contribution of beamlet $j$ to the total dose deposited in voxel $i$. The total dose received by voxel $i$ can be calculated as $\sum_{j=1}^{N} D_{i j} w_{j}$ with $w_{j}$ representing the intensity of beamlet $j$. For this particular case, the FMO model can then be defined as follows:

$$
\begin{aligned}
& f_{1}(w)=\operatorname{Min} \underset{i \in \text { Spinal cord }}{\operatorname{Max}} \sum_{j=1}^{N} D_{i j} w_{j} \quad \text { (1) } \quad f_{2}(w)=\operatorname{Min} \underset{i \in \operatorname{Brainstem}}{\operatorname{Max}} \sum_{j=1}^{N} D_{i j} w_{j} \\
& f_{3}(w)=\operatorname{Min} \frac{\sum_{i \in \text { Right parotid } j=1} \sum_{j=1}^{N} D_{i j} w_{j}}{\#\{i: i \in \text { Right parotid }\}}(3) \quad f_{4}(w)=\operatorname{Min} \frac{\sum_{i \in \text { Left parotid }} \sum_{j=1}^{N} D_{i j} w_{j}}{\#\{i: i \in \text { Left parotid }\}} \\
& f_{5}(w)=\operatorname{Min} \operatorname{Max}\left\{0,70-D_{95 \%}\left(P T V_{70}\right)\right\} \quad f_{6}(w)=\operatorname{Min} \operatorname{Max}\left\{0,59.4-D_{95 \%}\left(P T V_{59}\right)\right\}
\end{aligned}
$$

$$
f_{7}(w)=\operatorname{Min} V_{107 \%}\left(P T V_{70}\right)
$$


Subject to:

$$
\begin{aligned}
& \sum_{j=1}^{N} D_{i j} w_{j} \leq 45, \forall i \in \text { spinal cord } \quad \text { (8) } \quad D_{95 \%}\left(P T V_{70}\right) \geq 66.5 \\
& \sum_{j=1}^{N} D_{i j} w_{j} \leq 54, \forall i \in \text { brainstem } \quad \text { (10) } \quad \sum_{j=1}^{N} D_{i j} w_{j} \leq 74.9, \forall i \in P T V_{70}
\end{aligned}
$$

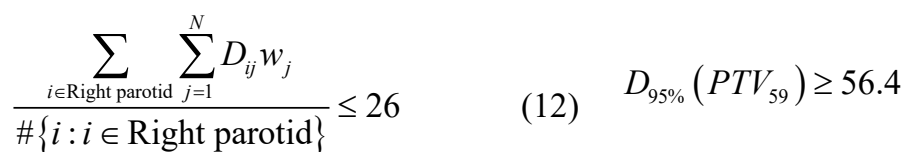

$$
\begin{aligned}
& \frac{\sum_{i \in \text { Left parotid }} \sum_{j=1}^{N} D_{i j} w_{j}}{\#\{i: i \in \text { Left parotid }\}} \leq 26 \quad \text { (14) } \quad \sum_{j=1}^{N} D_{i j} w_{j} \leq 74.9, \forall i \in P T V_{70}
\end{aligned}
$$

$V_{107 \%}\left(P T V_{59}\right) \leq$ Percentage of $\mathrm{PTV}_{70}$ volume inside $\mathrm{PTV}_{59}$ plus a $10 \%$ margin

$$
w_{j} \geq 0, j=1, \ldots, N
$$

\section{Heuristic procedure based on FIS}

In order to optimize this nonlinear multiobjective optimization problem, a much simpler problem will be iteratively solved. Let $U_{i}$ and $L_{i}$ be upper/lower bounds associated with voxel $i$. $\underline{\lambda}_{i}$ and $\bar{\lambda}_{i}$ are penalty weights. The FMO model is defined as:

$$
\begin{gathered}
f(w)=\operatorname{Min}_{w} \sum_{i=1}^{V}\left[\underline{\lambda}_{i} \max \left\{0, L_{i}-\sum_{j=1}^{N} D_{i j} w_{j}\right\}^{2}+\bar{\lambda}_{i} \max \left\{0, \sum_{j=1}^{N} D_{i j} w_{j}-U_{i}\right\}^{2}\right] \\
\text { s.t. } w_{j} \geq 0, j=1, \ldots, N
\end{gathered}
$$

The objective function considered does not have any clinical meaning whatsoever. This optimization problem will only be used as a tool for finding RT plans satisfying all the defined constraints. This problem will be iteratively solved, having its parameters (both weights and bounds) automatically changed resorting to FIS. Structures violating the respective constraints will have their importance increased in (18), either by changing the corresponding bounds, or weights, or both. Let $d_{S}$ represent the distance between the dosimetry values of the current solution and the bounds defined by 
the violated constraints for $S$. The fuzzy rules considered are of the form: if $d_{S}$ is large then increase (decrease) $L_{S}\left(U_{S}\right)$ by a large amount; if $d_{S}$ is medium then increase (decrease) $L_{S}\left(U_{S}\right)$ by a medium amount; if $d_{S}$ is small then increase (decrease) $L_{S}$ ( $U_{S}$ ) by a small amount, where concepts like small, medium or large are defined by fuzzy membership functions and the change in the right hand side of the constraint is determined by FIS. A detailed description of this procedure can be found in [1]. The algorithm tries to find a solution that satisfies all constraints. If this is not possible, then it will relax some of the constraints, also using FIS and considering the distance between each one of the dose metrics and the desired values (the greater the distance, the greater the relaxation, by changing the right hand side values of the constraints (8)-(16)). When a feasible solution is reached, the algorithm tries to improve this solution by being more demanding regarding the dosimetry constraints. The right hand side values are, once again, changed by using FIS. In [1] the multiobjective nature of the problem was not explicitly considered, and the planner is asked to assign priorities to all structures. These priorities would define (again using a FIS) how the right hand side values of the restrictions would be changed: the algorithm would give more importance to structures with higher priorities, meaning that it would be more demanding with these structures, accepting worse values in the other ones. The algorithm will stop when it is not possible to improve the current treatment plan further. In this work no priority list is considered and equal importance is given to all the structures. A set of solutions is calculated. The algorithmic approach is described next:

1. Choose a set of radiation beam angles. improve $\leftarrow 0$;

2. Initialize all the model's parameters; $i t \leftarrow 0$.

3. Solve the FMO with the current parameters; $i t \leftarrow i t+1$.

4. Do the dosimetry calculations. Admissible $\leftarrow$ true.

5. For each structure $S$

(a) If $S$ is violating a constraint then change the upper/lower bounds associated with $S$ according to FIS. Admissible $\leftarrow$ false.

(b) If the upper/lower bound associated with $S$ has reached a predetermined threshold, then change the corresponding weight according to FIS.

6. If Not Admissible go to 7, else go to 9 .

7. If $i t \leq N \max$ then go to 3 , else go to 8 .

8. If improve then go to 9. Else relax some of the violated constraints using FIS. $i t \leftarrow 0$. Go to 3 .

9. improve $\leftarrow 1$. For each structure $S$ and for each objective function $f$ involving $S$

(a) Change the right hand side of the constraint related with $S$ and $f$, by using FIS.

(b) Execute 2 to 8 .

(c) Save the current solution to a set SOL.

10. If every set of angles was already considered, then go to 11 . Else, select a different set of angles and go to 2 .

11. Analyse set $S O L$ and identify all the potential non-dominated solutions.

The algorithm begins by considering a given set of beam angles, and tries to find an admissible solution (steps 2 to 8 ). If it is not possible to find an admissible solu- 
tion, the algorithm relaxes some of the constraints (step 8). When a solution is finally calculated (step 9), then the algorithm will consider a structure $S$ and one objective function related with that structure at a time. The right hand side of the corresponding constraint will be more demanding (step 9.a). This is interpreted as a new problem, that is again solved by steps 2 to 8 . The procedure is repeated for all pairs of structures and objectives. All the solutions that are calculated along the process are saved. When all sets of beam angles have been tried, this pool of solutions is analyzed so that only the non-dominated solutions are kept. These solutions are non-dominated considering this set, but it is not possible to assure that they are indeed non-dominated for the original problem. This algorithm can thus be interpreted as a heuristic procedure that approximates the non-dominated Pareto frontier. Step 9a should be further explained. It is motivated by a well know result by Ross and Soland [22], where they show that it is possible to find non-dominated solutions for a linear multiobjective mixed integer programming problem by simply using a weighted objective function and additional constraints, one for each objective. Changing the right hand side of these constraints and optimizing the problem will lead to non-dominated solutions. Although we are not in the presence of a mixed integer linear multiobjective programming problem, the idea is the same: changing the right hand side of constraints that are related with the objective functions will trigger the discovery of new solutions. This change is done looking at how far the current solution is from the upper/lower bounds defined by the constraint associated with the objective function. Simple fuzzy rules are considered, assuming that if the current solution is fulfilling the current constraint by a large amount (the slack is high) the algorithm can be more demanding. On the contrary, if they are barely fulfilling the constraint, then the change has to be only slight.

After generating a set of non-dominated solutions, it is still possible that the planner wants to calculate other solutions different from the ones already available. It is possible to consider an interactive procedure where the planner chooses two known solutions. Bounds based on these two solutions and his preferences can be defined. To calculate this new solution, two different situations have to be considered: if both solutions were generated using the same beam angles set, then it is possible to simply consider a linear combination of the corresponding fluence maps $[12,23]$ to find a new admissible solution, taking no more than a few seconds of computational time. If they were generated considering two different sets of beam angles, then it is no longer possible to consider a linear combination of fluence maps directly. The algorithm will consider one beam angle set at a time and will look for a solution generated by that set that is as close as possible to the solution generated with the other beam angle set. A linear combination is then considered. This means that two new solutions are generated, one for each beam angle set. If at least one of the solutions satisfies the new bounds, then the solution is presented to the planner. If not, the algorithm has to be executed again, considering only the two beam angle sets and the new bounds. The computational time is expected to be in the order of 4 to 30 minutes (according to computational experiments made). Consider the example depicted in Table 2, where two solutions have been found, but the planner wants to calculate another one. The type of existing constraints will determine the new bounds to consider (where $\varepsilon \rightarrow 0$ ). 
Table 2. Calculating a new solution

\begin{tabular}{lllll}
\hline Structure & Type of constraint & Solution 1 & Solution 2 & New bounds \\
\hline Spinal cord & Maximum dose & 44.5 & 38.1 & $44.5-\varepsilon$ \\
Brainstem & Maximum dose & 53.7 & 51.3 & $53.7-\varepsilon$ \\
Left parotid & Mean dose & 21.9 & 20.9 & $21.9-\varepsilon$ \\
Right parotid & Mean dose & 21.9 & 22.5 & $21.9-\varepsilon$ \\
PTV $_{70}$ & $D_{95 \%}$ & 67.1 & 66.5 & $66.5+\varepsilon$ \\
PTV $_{70}$ & Maximum dose & 74.9 & 74.9 & 74.9 \\
PTV $_{59}$ & $D_{95 \%}$ & 57.5 & 56.9 & $56.9+\varepsilon$ \\
\hline
\end{tabular}

\section{Illustration of the application of the procedure}

The algorithm was applied to one head-and-neck cancer case where proper PTV coverage and OAR sparing was difficult to obtain in clinical practice (Fig. 1). The OARs and PTVs considered are defined in Table 1, as well as the medical prescription.

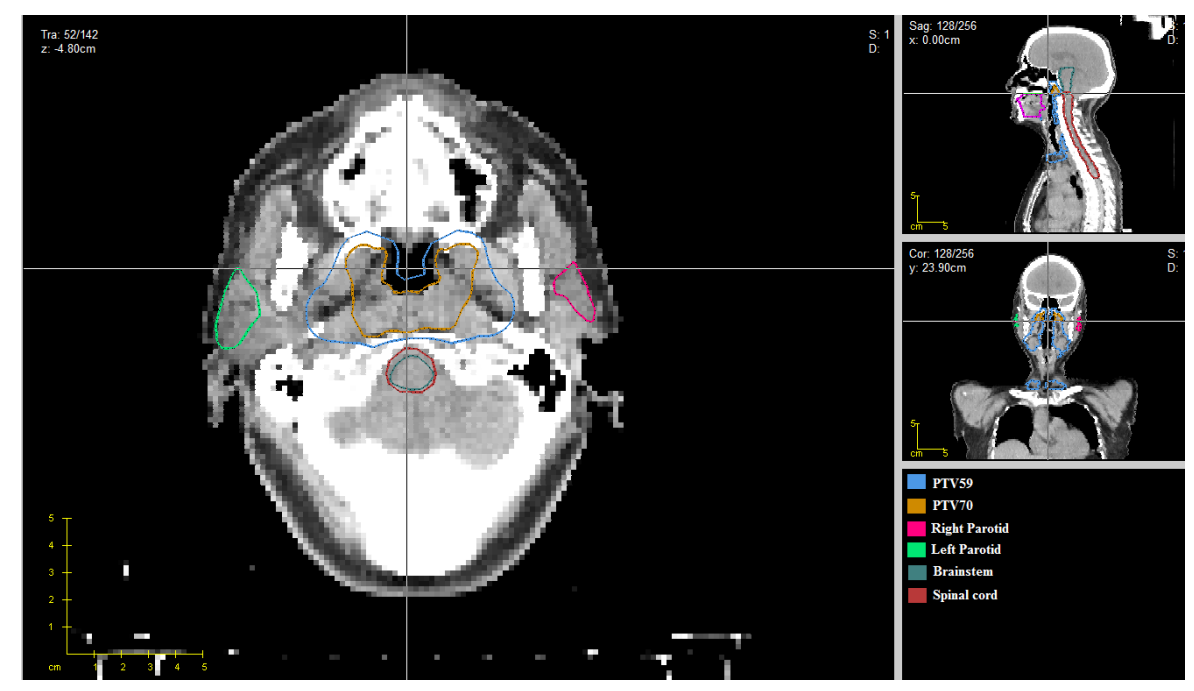

Fig. 1. Contoured structures in one CT slice for the considered case.

In clinical practice, most of the times, these cases are treated with 5 up to 11 beam angles. In this paper 9 beam angle plans are considered, and every equidistant beam angle solution with $5^{\circ}$ discretization was tried. Tests were performed on an Intel Core i7 CPU $2.8 \mathrm{GHz}$ computer with 4GB RAM and Windows 7. CERR 3.2.2 [24] and MATLAB 7.4.0 (R2007a) were used. The dose was computed using CERR's pencil beam algorithm (QIB), with corrections for heterogeneities. The sample rate for Body was 32 and for the remaining structures was 4 . The FMO problem was solved using a trust-region-reflective algorithm (fmincon). FIS made use of the Fuzzy Sets Toolbox. The algorithm was initialized as described in [1]. It found a total of 78 different potential non-dominated solutions in approximately 12 hours of computational time. 
Fig. 2 shows the distribution of the dosimetry values for each structure using boxplots. Fig. 3 shows a heatmap created by considering the dosimetry values of the solutions set. It can be seen that there are not many differences in the PTV coverage, and choices have to be made regarding the irradiation of parotids and spinal cord. Fig. 4 shows a line chart considering the subset of solutions that are in the quartile with higher doses delivered to PTVs. Dominated solutions would be represented by a line that would be always above at least one other line. It is possible to observe that they are all non-dominated between themselves.

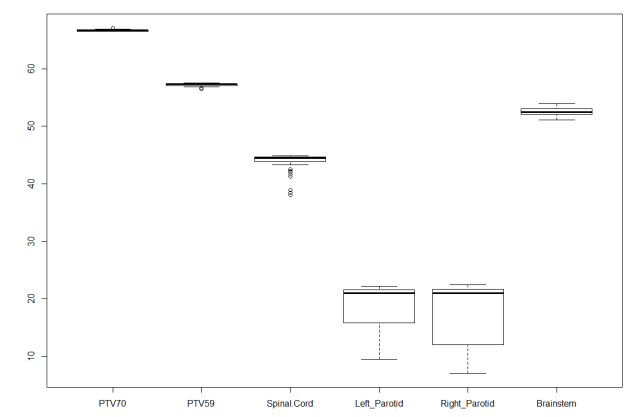

Fig. 2. Box-plot of the dosimetry values for each structure.

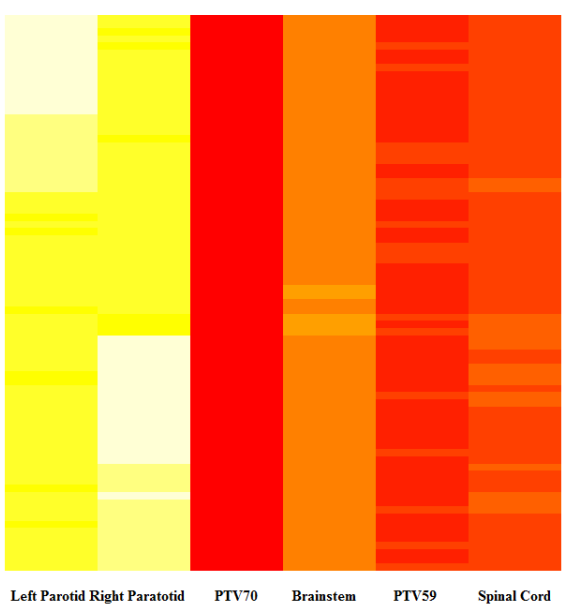

Fig. 3. Heatmap illustrating all the nondominated solutions found.

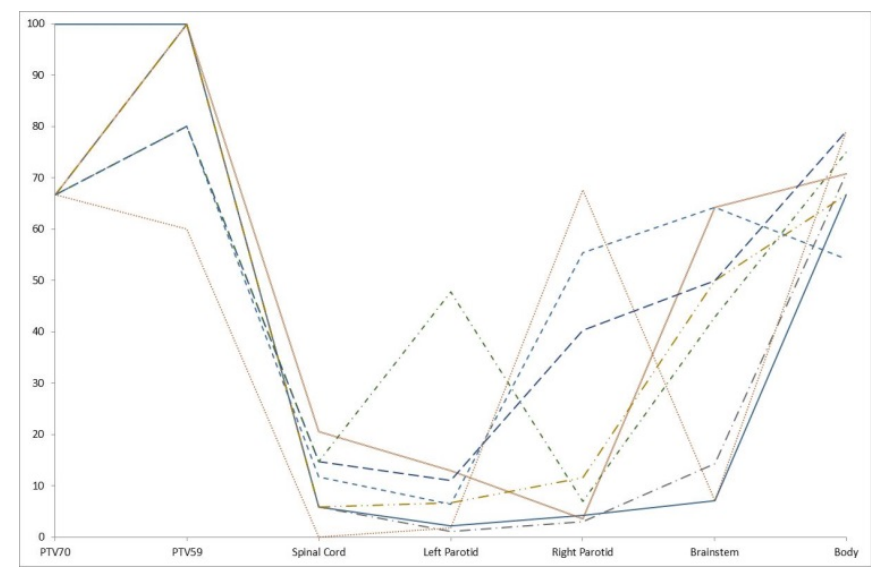

Fig. 4. Line chart considering dosimetry values scaled into [0-100] where 0 is the worst value for the structure and 100 is the best. 


\section{Conclusions}

In this paper a new methodology based on FIS that is able to calculate sets of nondominated solutions for RT planning is described. This set is built without requiring human intervention. The a priori calculation of this set could then support an interactive navigation procedure, where the planner can explore the existing tradeoffs and choose the best treatment plan according to his preferences. It is also possible to consider an interactive procedure, where new plans are calculated if the planner wants to explore new regions of the Pareto frontier. The optimal design of such a decision support system, and the exploration of new visualization tools that allow the user to simultaneously deal with more than three objectives, is out of the scope of this paper and is an interesting path of research. The analysis of the number of solutions calculated with each set of beam angles and the corresponding tradeoffs can also provide valuable insights for the integration of a proper beam angle optimization in a multiobjective framework.

\section{Acknowledgments}

This work has been supported by the Fundação para a Ciência e a Tecnologia (FCT) under project grant UID/MULTI/00308/2013.

\section{References}

1. Dias, J., Rocha, H., Ventura, T., Ferreira, B.,Lopes, M.d.C.: Automated fluence map optimization based on fuzzy inference systems. Medical physics. 43, 1083-1095 (2016)

2. Li, R.-P.,Yin, F.-F.: Optimization of inverse treatment planning using a fuzzy weight function. Medical physics. 27, 691-700 (2000)

3. Yan, H., Yin, F.-F., Guan, H.,Kim, J.H.: Fuzzy logic guided inverse treatment planning. Medical physics. 30, 2675-2685 (2003)

4. Yan, H., Yin, F.-F.,Willett, C.: Evaluation of an artificial intelligence guided inverse planning system: clinical case study. Radiotherapy and oncology. 83, 76-85 (2007)

5. Stieler, F., Yan, H., Lohr, F., Wenz, F.,Yin, F.-F.: Development of a neuro-fuzzy technique for automated parameter optimization of inverse treatment planning. Radiation Oncology. 4, 39 (2009)

6. Kierkels, R.G.J., Visser, R., Bijl, H.P., Langendijk, J.A., van't Veld, A.A., Steenbakkers, R.J.H.M.,Korevaar, E.W.: Multicriteria optimization enables less experienced planners to efficiently produce high quality treatment plans in head and neck cancer radiotherapy. Radiation Oncology. 10, 87 (2015)

7. Thieke, C., Kufer, K.H., Monz, M., Scherrer, A., Alonso, F., Oelfke, U., Huber, P.E., Debus, J.,Bortfeld, T.: A new concept for interactive radiotherapy planning with multicriteria optimization: First clinical evaluation. Radiotherapy and Oncology. 85, 292-298 (2007)

8. Romeijn, H.E., Dempsey, J.F.,Li, J.G.: A unifying framework for multi-criteria fluence map optimization models. Physics in Medicine and Biology. 49, 1991-2013 (2004) 
9. Craft, D., Halabi, T., Shih, H.A.,Bortfeld, T.: An approach for practical multiobjective IMRT treatment planning. International journal of radiation oncology* Biology* Physics. 69, 1600-1607 (2007)

10. Craft, D.L., Halabi, T.F., Shih, H.A.,Bortfeld, T.R.: Approximating convex Pareto surfaces in multiobjective radiotherapy planning. Medical physics. 33, 3399-3407 (2006)

11. Craft, D.,Richter, C.: Deliverable navigation for multicriteria step and shoot IMRT treatment planning. Physics in medicine and biology. 58, 87 (2013)

12. Craft, D.,Monz, M.: Simultaneous navigation of multiple Pareto surfaces, with an application to multicriteria IMRT planning with multiple beam angle configurations. Medical physics. 37, 736-741 (2010)

13. Teichert, K., Süss, P., Serna, J.I., Monz, M., Küfer, K.H.,Thieke, C.: Comparative analysis of Pareto surfaces in multi-criteria IMRT planning. Physics in medicine and biology. 56, $3669(2011)$

14. Holdsworth, C., Kim, M., Liao, J.,Phillips, M.H.: A hierarchical evolutionary algorithm for multiobjective optimization in IMRT. Medical physics. 37, 4986-4997 (2010)

15. Holdsworth, C., Kim, M., Liao, J.,Phillips, M.: The use of a multiobjective evolutionary algorithm to increase flexibility in the search for better IMRT plans. Medical physics. 39, 2261-2274 (2012)

16. Aubry, J.-F., Beaulieu, F., Sevigny, C., Beaulieu, L.,Tremblay, D.: Multiobjective optimization with a modified simulated annealing algorithm for external beam radiotherapy treatment planning. Medical physics. 33, 4718-4729 (2006)

17. Breedveld, S., Storchi, P.R.M., Voet, P.W.J.,Heijmen, B.J.M.: iCycle: Integrated, multicriterial beam angle, and profile optimization for generation of coplanar and noncoplanar IMRT plans. Medical physics. 39, 951-963 (2012)

18. Deasy, J.O.: Multiple local minima in radiotherapy optimization problems with dosevolume constraints. Medical Physics. 24, 1157 (1997)

19. Zarepisheh, M., Shakourifar, M., Trigila, G., Ghomi, P.S., Couzens, S., Abebe, A., Noreña, L., Shang, W., Jiang, S.B.,Zinchenko, Y.: A moment-based approach for DVH-guided radiotherapy treatment plan optimization. Physics in medicine and biology. 58, 1869-1887 (2013)

20. Scherrer, A., Yaneva, F., Grebe, T.,Küfer, K.-H.: A new mathematical approach for handling DVH criteria in IMRT planning. Journal of Global Optimization. 1-22 (2014)

21. Romeijn, H.E., Ahuja, R.K., Dempsey, J.F.,Kumar, A.: A new linear programming approach to radiation therapy treatment planning problems. Operations Research. 54, 201$216(2006)$

22. Ross, T.,Soland, R.: A Multicriteria Approach to the Location of Public Facilities. European Journal of Operational Research. 4, 307-321 (1980)

23. Hoffmann, A.L., Siem, A.Y.D., den Hertog, D., Kaanders, J.H.A.M.,Huizenga, H.: Derivative-free generation and interpolation of convex Pareto optimal IMRT plans. Physics in medicine and biology. 51, 6349 (2006)

24. Deasy, J.O., Blanco, A.I.,Clark, V.H.: CERR: A computational environment for radiotherapy research. Medical Physics. 30, 979-985 (2003) 